\title{
Les statues habillées dans le catholicisme. Entre histoire de l'art, histoire religieuse et anthropologie
}

À propos de : PAGNOZZATo Riccarda (a cura di), Madonne della laguna. Simulacri "da vestire" dei secoli XIV-XIX (Vierges de la lagune. Statues «à habiller » du XIV ${ }^{\mathrm{e}}$ au XIX ${ }^{\mathrm{e}}$ siècle), Roma, Istituto della Enciclopedia italiana, 1993, $381 \mathrm{p}$.

PAGNOZZATO Riccarda, " Le donne delle vestizioni e i simulacri mariani » (Les satues de la Vierge et les femmes qui les habillent) in Silvestrini Elisabetta, Gri Giampaolo, Pagnozzato Riccarda, Donne, Madonne, Dee. Abito sacro e riti di vestizione, gioiello votivo, "vestritici" : un itinerario antropologico in area lagunare veneta (Femmes, Madonnes, Déesses. Vêtement sacré et rites d'habillage, bijou votif, « habilleuses » : un itinéraire anthropologique dans l'aire lagunaire de la Vénétie). Padova, Il Poligrafo, 2003, p. 99-286.

GENOVESE Valeria, Statue vestite e snodate. Un percorso (Statues habillées et articulées. Un parcours). Pisa, Scuola Normale Superiore, 2011, 527 p. DuRAND Maximilien (dir.), Icône de mode, catalogue d'exposition, EMCC, Lyon, 2011, $224 \mathrm{p}$.

\section{Marlène Albert Llorca}

\section{(C) OpenEdition} Journals

\section{Édition électronique}

URL : http://journals.openedition.org/assr/25380

DOI : $10.4000 /$ assr. 25380

ISSN : $1777-5825$

Éditeur

Éditions de l'EHESS

\section{Édition imprimée}

Date de publication : 30 décembre 2013

Pagination : 11-23

ISSN : 0335-5985 
Ce document a été généré automatiquement le 19 avril 2019

(c) Archives de sciences sociales des religions 


\section{Les statues habillées dans le catholicisme. Entre histoire de l'art, histoire religieuse et anthropologie}

À propos de : PAGNOZZATo Riccarda (a cura di), Madonne della laguna. Simulacri "da vestire" dei secoli XIV-XIX (Vierges de la lagune. Statues « à habiller » du XIV ${ }^{\mathrm{e}}$ au XIX ${ }^{\mathrm{e}}$ siècle), Roma, Istituto della Enciclopedia italiana, 1993, $381 \mathrm{p}$.

PAGNOZZATo Riccarda, « Le donne delle vestizioni e i simulacri mariani » (Les satues de la Vierge et les femmes qui les habillent) in Silvestrini Elisabetta, Gri Giampaolo, Pagnozzato Riccarda, Donne, Madonne, Dee. Abito sacro e riti di vestizione, gioiello votivo, "vestritici" : un itinerario antropologico in area lagunare veneta (Femmes, Madonnes, Déesses. Vêtement sacré et rites d'habillage, bijou votif, « habilleuses » : un itinéraire anthropologique dans l'aire lagunaire de la Vénétie). Padova, Il Poligrafo, 2003, p. 99-286.

GENOVESE Valeria, Statue vestite e snodate. Un percorso (Statues habillées et articulées. Un parcours). Pisa, Scuola Normale Superiore, 2011, 527 p.

DURAND Maximilien (dir.), Icône de mode, catalogue d'exposition, EMCC, Lyon, 2011, $224 \mathrm{p}$.

\section{Marlène Albert Llorca}

À partir des années 1990, on a vu se multiplier, en Italie en particulier, les ouvrages consacrés à une pratique dévotionnelle répandue dans tout le monde catholique depuis la fin du Moyen Âge, mais dont les historiens, historiens de l'art et anthropologues du christianisme ont longtemps méconnu l'importance: l'usage d'habiller les statues de vêtements de tissu. Condamné par les historiens de l'art parce qu'il masque les sculptures 
que recouvrent robes et manteaux, il a également été rejeté par les religieux qui y voient une pratique idolâtre ou, au contraire, profanatrice - l'habillement et la parure des statues, celles de la Vierge notamment, étant parfois jugée «indécente ». À la fin du XIX ${ }^{\mathrm{e}}$ siècle et au cours du suivant, on va donc dénuder nombre de ces statues ou mettre au rebut les mannequins en bois qui tenaient lieu de statues dans certaines églises.

Un ouvrage collectif dirigé par une artiste peintre, Riccarda Pagnozzato, révèle en 1993 l'importance de ce type d'images cultuelles. Il recense, sur Venise et les îles de sa lagune, cinquante-six Vierges habillées ${ }^{1}$ dont les supports (statue ou mannequin) datent du XIV ${ }^{\mathrm{e}}$ siècle pour les plus anciens et du XVIII ${ }^{\mathrm{e}}$ pour les plus récents. L'ouvrage donne aussi des éléments, issus à la fois des enquêtes ethnographiques que R. Pagnozzato avait commencées seule dès les années 1970 et d'un minutieux dépouillement des archives diocésaines, permettant d'en saisir les significations sociales et religieuses. Ce remarquable travail n'est pas resté sans suite. Au cours de la décennie qui suit sa publication, plusieurs inventaires du même type sont entrepris, souvent en lien avec la réalisation d'une exposition, dans d'autres provinces d'Italie : Bari, dans les Pouilles, en 1995, Arezzo (Toscane) et Pérouse (Ombrie) en 2005, Bologne (Emilie-Romagne) en 2008, Latina, dans le Latium, en $2010^{2}$. Dans la même période, paraissent aussi quantité d'articles écrits par des historiens de l'art, des restaurateurs, des anthropologues qui travaillent sur les traditions populaires italiennes (cf. Jacquard 2009, Silvestrini 2010b, Bortolotti 2011). Certains de ces auteurs ont été formés à la fois à l'histoire de l'art et à l'anthropologie et ont travaillé dans des musées d'arts et traditions populaires. Ainsi d'Elisabetta Silvestrini, qu'un intérêt ancien pour les poupées - poupées « de fécondité " offertes aux jeunes mariées ou poupées religieuses (cf. Silvestrini e Simeoni 1987) - avait sensibilisée à la question de l'habillage de figures anthropomorphes: auteur de l'inventaire des statues habillées du Latium (des Vierges, mais aussi des saints et des Enfants Jésus), elle a aussi écrit plusieurs articles sur ce thème et dirigé un numéro récent de la revue Errefe. La ricerca folklorica (2010b).

3 Je m'arrêterai très inégalement sur cet ensemble de publications qui présente, à mes yeux, deux intérêts. Il contribue, d'une part, à manifester la complexité des facteurs qui suscitent (ou pas) un processus de patrimonialisation. Il a aussi et surtout l'intérêt de mettre en évidence la porosité des frontières entre histoire de l'art, histoire religieuse et anthropologie dès lors qu'il s'agit d'étudier les images, et notamment les images cultuelles. De ce point de vue, l'ouvrage qu'une historienne de l'art, Valeria Genovese, vient de publier sur les statues habillées et articulées retient particulièrement l'attention. Inscrit dans la lignée des travaux d'historiens de l'art comme Hans Belting et David Freedberg qui ont affirmé la nécessité d'étudier «toutes les images, et pas seulement celles que l'on considère comme artistiques ", il intéresse non seulement les historiens de l'art, mais aussi les anthropologues et les historiens du fait religieux ${ }^{3}$.

\section{Des valorisations patrimoniales inégales}

4 Le foisonnement de publications que je viens d'évoquer est sans nul doute une des manifestations de la «fièvre patrimoniale » qui caractérise notre époque. Tout, « de la cathédrale à la petite cuillère »" étant désormais jugé digne d'être conservé, on conçoit qu'on ait fini par se préoccuper du destin des "statues ${ }^{5}$ habillées et de leur garde-robe, souvent menacées de disparaître. R. Pagnozzato découvrit ainsi une Vierge du Rosaire dans le grenier de l'église de Chioggia où on l'avait reléguée, sans doute au début du xx 
siècle (1993: 296). La "fièvre patrimoniale», cependant, ne s'applique pas uniformément, comme le montre la différence d'intérêt porté aux « statues » à habiller et à leurs vêtements en Italie et en France.

Un seul ouvrage, à ma connaissance, a été entièrement consacré dans notre pays à ce type d'objets ou, plus précisément, à l'habillement des «statues » de la Vierge ${ }^{6}$, le catalogue d'une exposition présentée en 2011-2012 au Musée des tissus de Lyon sous un titre quelque peu provocateur : «Icône de mode ». Maximilien Durand, directeur du musée et commissaire de l'exposition, conçut ce projet en découvrant une initiative tout à fait singulière. En 2008, quelques paroissiens et paroissiennes de la Daurade, dont l'église est consacrée à une Vierge noire très vénérée à Toulouse et dans sa région, décidèrent de créer une Association pour la Promotion du Patrimoine de la Daurade (APPD). Ladite Vierge - qui est une «statue » à habiller - disposait alors de plusieurs jeux de vêtements, fort beaux pour la plupart, mais aussi en piteux état. Deux des membres de l'association décidèrent de solliciter les propriétaires de magasins de confection de la ville et des professionnels de la Haute Couture française en leur proposant d'offrir une nouvelle robe à la Vierge. Répondirent à l'appel, à Toulouse, la propriétaire d'une boutique de mariage et des enseignants d'un lycée professionnel consacré aux métiers de la mode et, à l'extérieur, quelques grands couturiers: Jean-Charles de Castelbajac, Franck Sorbier, Jean-Michel Broc. Dans l'exposition présentée à Lyon figuraient à la fois les robes que les uns et les autres avaient offertes à la Vierge et quelques-unes de ses anciennes robes, dûment restaurées par le musée.

Le succès de l'initiative de l'APPD a sans doute dépassé les espérances de ses promoteurs. Il reste qu'elle émanait de quelques individus (des femmes très impliquées dans la vie de l'Église), et non pas d'organismes publics comme le sont les services patrimoniaux qui ont pris en charge, en Italie, l'inventaire, la restauration et la conservation des images à habiller et de leurs vêtements. On peut y voir un indice de l'inégale valeur patrimoniale conférée aux objets religieux, en France et en Italie ${ }^{7}$, du moins à ceux qui ne sont pas qualifiés comme œuvres d'art par les tenants de la « culture légitime».

\section{Un regard d'artiste}

7 À la différence de la plupart des ouvrages cités précédemment, le livre dirigé par R. Pagnozzato n'est pas seulement un inventaire; il contient aussi des études qui s'attachent à restituer, d'une part, l'histoire des Vierges à habiller et de leur habillement et, d'autre part, le sens que les fidèles confèrent au fait d'habiller la Vierge. Mentionnons seulement, pour faire bref, le fait qu'elle porte des vêtements qu'ils lui ont donnés, parfois après les avoir eux-mêmes portés. Le vêtement institue ainsi un lien entre les fidèles et la «statue ", tout en contribuant à « animer " celle-ci. L'apport essentiel de l'ouvrage est, cependant, d'avoir révélé la valeur conférée à l'acte d'habiller la statue, opération à laquelle personne, à l'exception de l'historien R. Trexler', ne s'était intéressé jusque-là. Toutes les Vierges, en effet, disposent au minimum de deux jeux de vêtements, ce qui permet de les changer au moins une fois l'an pour leur fête. L'opération est accomplie par quelques femmes de la localité qui officient à huis clos. Elles seules, donc, peuvent voir ou toucher le «corps » de la Vierge, expérience qu'elles décrivent toujours avec une très vive émotion, où il entre à la fois de la crainte, de la fierté, du respect et la tendresse que l'on éprouve pour un proche bien aimé (Pagnozzato $2003: 174,182,200)^{9}$. 
Dans le second ouvrage auquel elle a collaboré, Donne, Madonne, Dee, R. Pagnozzato explique que son intérêt pour les Vierges habillées a été suscité par l'expérience qu'elle a faite - par hasard et, en quelque sorte, par effraction - de l'habillage d'une statue. Alors qu'elle était dans une église des Pouilles au moment de la Semaine sainte, quelques femmes de la confrérie de la Vierge des Douleurs y entrèrent et, ne s'étant pas aperçues de sa présence, elles se mirent à changer la statue après avoir fermé les portes de l'église (R. Pagnozzato 2003 : 199-200). Frappée par l'intensité émotionnelle du rite, elle décida d'enquêter sur les Vierges habillées et les modalités de leur habillage dans la région de Venise, où elle réside.

R. Pagnozzato a exposé le résultat de ses enquêtes auprès des habilleuses des Vierges vénitiennes dans son second livre et non pas dans Madonne della laguna. Toutefois, la question de la relation des habilleuses à la «statue» dont elles s'occupent n'est pas absente de ce premier travail: elle l'aborde à travers ses photographies des Vierges inventoriées. À la différence de celles que l'on trouve dans les catalogues d'œuvres d'art, ces photographies n'ont pas seulement un caractère informatif. Comme le précise en effet R. Pagnozzato dans l'introduction du livre: «La photographie a été conçue comme un instrument de recherche et un outil pour communiquer visuellement les significations sociales, religieuses et humaines portées par les statues: une confrontation continuelle entre l'œil et l'émotion» (1993: 26). Cette émotion est surtout sensible dans les photographies des Vierges dénudées. Cela peut sembler paradoxal, ces « statues » étant le plus souvent des mannequins dont on a façonné uniquement le haut du corps, le bas se réduisant à un châssis formé d'une pièce de bois à peine dégrossie ou de quelques liteaux. R. Pagnozzato, il est vrai, a pris surtout leur visage, et ces visages sont d'autant plus poignants ${ }^{10}$ que ces Vierges ont souvent un crâne dénudé, étant destinées non seulement à être habillées, mais aussi à être coiffées d'une perruque. Mais l'émotion est aussi grande lorsqu'on voit la Vierge du Rosaire de l'église de Chioggia - un mannequin articulé couchée sur le sol du grenier où on l'avait reléguée, les jambes percées de baguettes de bois et les bras levés comme si elle demandait pitié. (ill. 1 et 2). 


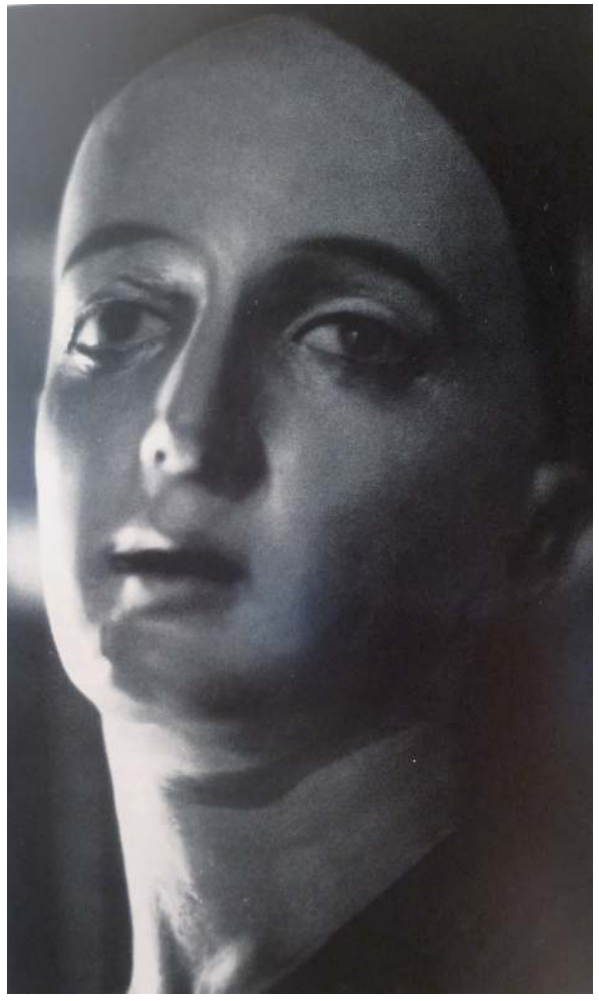

Vierge des douleurs, Vénise, église de S. Marcuola (photo R. Pagnozzato).

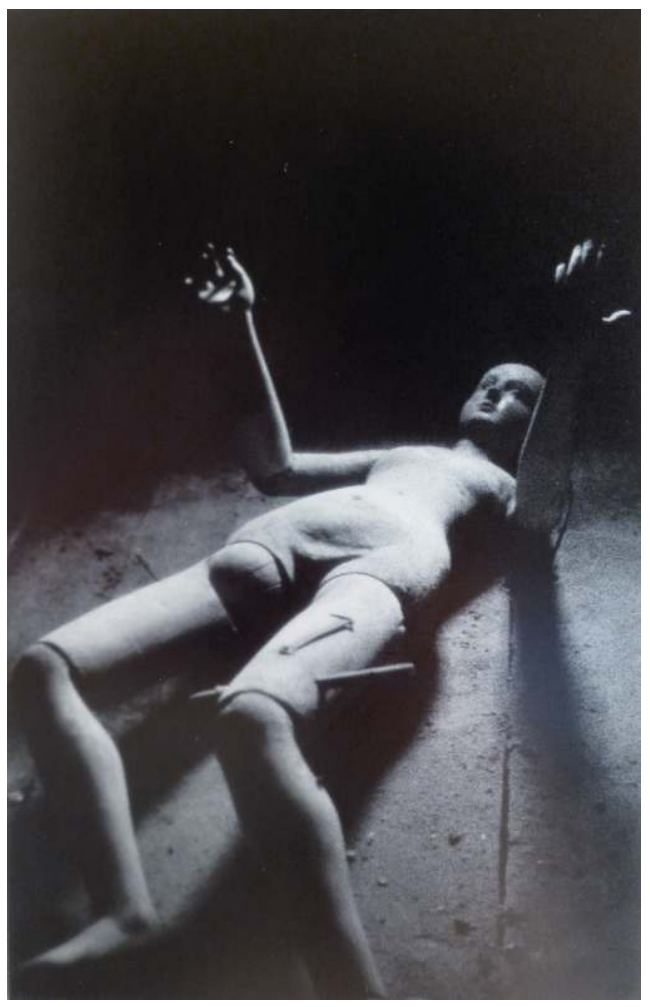

Vierge du Rosaire, Chioggia, église de Saint-Dominique (photo R. Pagnozzato). Nous remercions R. Pagnozzato et le comité éditorial de « Madonne della Laguna » d'avoir autorisé la publication des deux photos incluses dans cet article.

R. Pagnozzato n'est pas une universitaire, mais, comme je l'ai déjà indiqué, une artiste peintre. C'est bien parce qu'elle a fréquenté l'art $d u x^{e}$ siècle (songeons aux œuvres de 
Giorgio de Chirico ou de Hans Bellmer) qu'elle a pu reconnaître une valeur esthétique à des artefacts que l'on avait, au mieux, classés dans l'art populaire. C'est, par ailleurs, parce qu'elle utilise la photographie comme un médium artistique qu'elle parvient à communiquer, à travers elle, l'émotion suscitée par ces «statues ». Cette émotion est sans doute celle qu'elle a ressentie en les voyant. Mais c'est aussi celle de leurs habilleuses. Aucune d'entre elles, en effet, ne semble voir (ou ne veut voir) que la Vierge est un pauvre mannequin de bois; toutes s'accordent à dire que « leur » Vierge n'est pas pour elles une chose, mais une personne. C'est cette " humanité » que traduisent les photographies de R. Pagnozzato et c'est pourquoi ce ne sont pas seulement des illustrations. Elles ont, au contraire, fortement contribué, à mon sens, à la prise de conscience que les statues à habiller et ce qu'elles représentent pour les femmes méritaient considération.

\section{De « l'histoire de l'art » à « l'histoire de la piété »}

11 Artiste peintre, R. Pagnozzato a su se faire historienne et surtout ethnographe pour écouter et donner à entendre les femmes parlant de leur rapport aux Vierges habillées. Historienne de l'art, Valeria Genovese aborde les «statues habillées et articulées » sous un angle qui rejoint plusieurs des questionnements anthropologiques sur les images de culte.

Couvrant une période très large (de la fin du Moyen Âge au xix ${ }^{e}$ siècle) son livre porte à la fois, comme l'indique son titre, sur les statues habillées de vêtements de tissu et sur les statues articulées. Employer le terme statue dans les deux cas semble aujourd'hui abusif et c'est pourquoi je l'ai mis jusqu'ici entre guillemets. Selon V. Genovese cependant, cette extension du terme fut courante jusqu'au début du $\mathrm{xVIII}^{\mathrm{e}}$ siècle, période où les commanditaires commencent à préciser quel type d'objet ils veulent: une sculpture achevée, à laquelle on va désormais réserver le terme statue, ou un mannequin, articulé ou non $(2011: 148)^{11}$. Une statue, au sens actuel du terme, n'a pas besoin d'être habillée de vêtements de tissu (sauf s'il s'agit d'un nu, mais la chose est assez rare dans la statuaire religieuse) alors qu'une effigie articulée en porte nécessairement: le but de ce type d'artefact, du moins quand il a une destination religieuse, n'est pas de donner à voir l'ingéniosité de son mécanisme, mais de donner l'illusion de la vie, cela impliquant que le mécanisme qui permet de la produire soit dissimulé (op. cit. : 170-172).

V. Genovese souligne que la volonté de faciliter l'habillage ne suffit pas à expliquer l'existence d'effigies articulées. Cette motivation existe: une commande d'une Vierge de l'Annonciation passée par une communauté religieuse féminine de Sienne en 1523 précise ainsi que les bras doivent être faits « de telle sorte que l'on puisse l'habiller facilement » ( op. cit. : 143). Mais l'idée de construire ce type de figurations a aussi été suscitée par la place accordée à des formes liturgiques théâtralisées, voire au théâtre sacré proprement dit, dans le catholicisme prétridentin.

On peut classer dans le premier groupe les manipulations des crucifix pendant la Semaine sainte et plus précisément le Vendredi saint. Pour commémorer la mort du Christ, dans certaines églises, on descendait la statue représentant son corps de la croix où elle était attachée puis on la déposait dans un tombeau; dès le XIV ${ }^{\mathrm{e}}$ siècle, on dote certains crucifix de bras articulés pour faciliter cette déposition (op. cit. : 157). Plus connue, et encore en usage dans plusieurs régions d'Espagne et d'Italie (op.cit. : 160) est la mise en scène rituelle de la rencontre, à l'aube du dimanche de Pâques, de la Vierge et de son fils 
ressuscité. Elle se déroule sur une place de la localité où l'on amène, par des rues différentes, la statue du Christ et celle de la Vierge. Au moment où elles se rejoignent, on retire le voile noir dont on avait recouvert la statue de la Vierge, qui apparait alors toute de blanc vêtue ; en même temps, on l'incline vers la statue du Christ : la Vierge, souvent un mannequin articulé au niveau de la taille, salue ainsi son Fils. V. Genovese cite également plusieurs cas de théâtre sacré - un Jeu de saint Georges du $\mathrm{xV}^{\mathrm{e}}$ siècle par exemple - où interviennent à la fois des effigies, bien souvent des statues de bois polychrome, et des acteurs (op. cit. : 162-164).

On aperçoit, à travers cette brève évocation des pages consacrées au théâtre rituel, la richesse documentaire de l'ouvrage. Elle est d'autant plus grande qu'il comporte aussi une centaine de pages de transcription de textes d'archives ou de diverses publications (recueil de miracles, textes littéraires, etc.) et un riche dossier iconographique (cent trente photos en noir et blanc, onze en couleurs). L'intérêt essentiel de ce travail réside cependant, selon moi, dans la perspective adoptée et l'originalité de certaines des hypothèses de l'auteur.

V. Genovese souligne, dans son introduction, la complexité, voire l'impossibilité de « reconstruire [...] le regard que l'on portait sur ces œuvres » (op. cit. : 21). Mais, comme elle ajoute aussitôt que ce projet est " décidément intéressant ", on comprend que c'est bien lui qu'elle entend au moins ébaucher. Son but, en d'autres termes, n'est pas uniquement d'inventorier les œuvres, de les situer dans le temps, de déterminer leur style, leurs techniques de fabrication, leurs auteurs et, enfin, leurs restaurateurs - car les statues ont été bien souvent retouchées pour faciliter leur habillage. L'objectif est aussi de dire comment elles ont été perçues, d'une part par les historiens de l'art et, d'autre part, par les fidèles. Cela, en les prenant telles qu'elles apparaissaient dans les églises, c'est-àdire habillées et coiffées. Comme elle l'écrit avec humour :

«[...] il a fallu maitriser la frénésie de lever les jupes de toutes les statues "laides", frénésie fondée sur la conviction que, sous les vêtements et les perruques de bon nombre d'images cultuelles, se cachent des sculptures anciennes et de grande valeur. Il n'en va pas toujours ainsi et, si l'on n'estime pas comme il le faut la particularité de ce type d'images, on risque de perdre, en poursuivant un objet supposé relever de l'histoire de l'art, un objet relevant de façon certaine de l'histoire de la piété » (op. cit. : 24).

17 Le point de vue de l'historien de l'art n'est cependant pas évacué. V. Genovese s'arrête tout d'abord sur le silence de ce fondateur de l'histoire de l'art qu'est Giorgio Vasari à propos des «sculptures composites» étudiées: composites, à la fois parce que la sculpture proprement dite conjoint divers matériaux (par exemple, un corps en bois avec une tête et des mains en terre cuite vernissée ou en ivoire) et qu'elle est habillée, parée de bijoux, coiffée d'une perruque de cheveux, naturels ou pas. Vasari, donc, ne mentionne guère ce type de «statues » et ce, parce que son idéal esthétique, en matière de sculpture, est celui de la Grèce classique - ou plutôt de ce qu'on en connaît à la Renaissance : des statues en marbre ou, à défaut de marbre, des statues faites dans un seul matériau et monochromes (op. cit. : 104).

Le point de vue des historiens de l'art et plus généralement des lettrés du XIX ${ }^{e}$ siècle est un peu différent. La découverte, à la fin du XVIII ${ }^{\mathrm{e}}$ siècle, que les Anciens peignaient et habillaient leurs statues a en effet amené certains d'entre eux, sinon à réviser leur jugement esthétique sur les statues polychromes et, moins encore, sur les "statues" habillées, du moins à reconnaitre leur valeur dévotionnelle. Car, à partir du milieu du XIX ${ }^{\mathrm{e}}$ 
siècle, religieux et historiens relèvent souvent l'écart entre la qualification religieuse et esthétique des statues, en notant que les plus belles - selon les critères de l'esthétique savante - ne sont pas les plus vénérées, au contraire. Ce qui qualifie une statue comme miraculeuse, c'est bien souvent sa facture archaïque, signe de son ancienneté et, peut-on ajouter avec David Freedberg, son étrangeté ${ }^{12}$. Ont ainsi été ressenties comme "primitives ", à partir de l'époque moderne, les Vierges noires ou les statues en bois polychrome d'époque romane (op. cit. : 209 et suiv.). Les lettrés du XIXe siècle soulignent aussi que les fidèles valorisent bien moins la sculpture proprement dite que ses vêtements et ses parures, cela permettant de comprendre que l'on ait souvent mutilé les statues pour pouvoir les vêtir plus aisément (op. cit. : 26-27) ${ }^{13}$. Vêtements et parures manifestent en effet le caractère miraculeux de la statue (si on lui offre une parure, c'est que l'on a obtenu une grâce) renforçant, par là même, la dévotion que l'on a pour elle.

Il me semble utile de préciser que cette valorisation s'exprime aussi en termes esthétiques. C'est du moins ce qui ressort des terrains que j'ai réalisés en Espagne et dans les Pyrénées françaises, où l'on m'a toujours parlé de la beauté de la Vierge locale - celle que l'on vénère par-dessus tout. Cette "beauté » était visiblement liée au premier chef, pour mes interlocuteurs, à la richesse des parures de la statue, ce que relève aussi V. Genovese dans ses sources en ajoutant que c'est toujours la richesse des tissus utilisés, bien plus que leur décor ou la finesse de leurs broderies, que mettent en avant les textes sur les vêtements des statues (op.cit.: 213-215). Notons au passage que la hiérarchie établie par les fidèles entre les vêtements et la sculpture rejoint l'indifférence des commanditaires de la période prémoderne à l'égard de la distinction entre une statue au sens actuel du terme et un mannequin, articulé ou non. Pour les uns comme pour les autres, peu importe, en un sens, ce qu'il y a sous les vêtements. Le désaccord avec les historiens de l'art et les conservateurs des $\mathrm{XIX}^{\mathrm{e}}$ et $\mathrm{XX}^{\mathrm{e}}$ siècles est patent, ceux-ci ne cessant au contraire de réclamer que l'on arrête d'habiller les statues, à la fois parce que cet usage les abîme et parce qu'il empêche de voir la sculpture (op. cit. : 219-22).

Si V. Genovese a pris soin de relever ce que disent ses sources du regard porté par les fidèles sur les statues habillées, c'est qu'il est déterminé par la question de leur efficacité. Or, comme D. Freedberg, et comme les anthropologues, V. Genovese accorde une grande attention à cette dimension de l'image cultuelle. Pour rendre compte de ce qui lui confère cette efficacité, elle invoque, d'une part, ses caractéristiques formelles. J'ai déjà mentionné l'importance qu'elle accorde, après Freedberg, à son archaïsme, réel ou supposé. Elle évoque aussi son réalisme: yeux de verre enchâssés, habillement, mouvement, qui concourent à créer l'illusion que cet objet est une personne. Aussi importantes, pour un anthropologue, ses manipulations rituelles: ainsi de la pratique consistant à la dissimuler derrière une tenture qui n'est levée qu'à certaines dates, la faisant «apparaître» (op.cit.: 37-76). Un travail appuyé sur des enquêtes ethnographiques insisterait sans doute davantage sur l'importance du rite dans la construction de son efficacité, ainsi du secret qui entoure son habillage, mais les sources historiques sont sans doute muettes sur ce point.

21 Le questionnement sur les avatars de la perception des statues habillées me semble également rejoindre les préoccupations des historiens et des anthropologues - du moins de ceux qui jugent nécessaire de rendre compte des variations culturelles et pas seulement des invariants. Me semble particulièrement intéressante, de ce point de vue, la mise en évidence de l'évolution sémantique du terme «statue » et du fait qu'elle traduit 
une conception normative de ce que doit être l'artefact ainsi nommé : une figure achevée et sculptée dans un seul matériau.

\section{Conclusion}

Les travaux que je viens d'évoquer sont autant de pièces à verser au dossier, déjà conséquent, des croisements entre les questionnements des anthropologues et ceux des historiens de l'art ${ }^{14}$. Ceux-ci, comme on a pu le voir, ont considérablement élargi leur regard, ce qui les a conduits à soulever des questions très proches de celles que s'étaient posées les anthropologues. Ces derniers, à l'inverse, ont tout à gagner à prêter attention à ce qu'écrivent les historiens de l'art. Nombre de travaux publiés dans les dernières décennies, ceux de Bruno Latour au premier chef, ont conduit à réviser l'opposition entre les sujets humains, seuls capables d'agir de façon intentionnelle, et des objets qui subissent les effets de la volonté des premiers. Cette remise en cause rejoint, dans une certaine mesure, les analyses de l'anthropologue Alfred Gell sur l'agency des œuvres d'art, qu'il identifie comme étant des objets efficaces (2009). Or, cette question de l'efficacité est évidemment centrale lorsqu'on s'interroge sur les images cultuelles. Comme l'a montré Jérôme Baschet pour le Moyen Âge, elles ne valent pas seulement par leur contenu représentatif, mais par leur «objectalité»: l'image est située dans un lieu donné, déplacée à certaines dates, ornée de parures, et c'est ce qui la constitue et lui donne son efficacité. Cela impose de prendre connaissance de ce que savent les historiens de l'art et ceux qui sont formés à cette discipline - conservateurs et restaurateurs, mais aussi artistes comme R. Pagnozzato. Tous, en effet, ont acquis une sensibilité à l'objet et un savoir sur l'objet qui me semblent extrêmement précieux dès lors que l'on s'interroge sur les pouvoirs d'images qui sont aussi des objets.

\section{BIBLIOGRAPHIE}

ALBERT LLORCA Marlène, 2002, Les Vierges miraculeuses. Légendes et rituels, Paris, Gallimard, coll. « Le temps des images ».

ANDRIANO CESTARI Beatrice (dir.), 1995, Madonne vestite, catalogo della mostra, Giovinazzo.

BASCHET Jérôme, 1996, "Introduction : l'image-objet », in Baschet J. et Schmitt J.-C. : L'image. Fonctions et usages des images dans l'occident médiéval, Paris, Cahiers du Léopard d'Or.

BELTING Hans, 1998, Image et culte. Une histoire de l'image avant l'époque de l'art, Paris, Éditions du Cerf, coll. « Cerf Histoire » (Éd. Or, 1990).

BORTOLOTTI Lidia (a cura di), 2011, Vestire il sacro. Percorsi di conoscenza, restauro e tutela di Madonne, Bambini e Santi abbigliati, Istituto per i beni artistici, culturali e naturali della regione EmiliaRomagna, Bologna, Editrice Compositori.

FREEDBERG David, 1998, Le pouvoir des images, Paris, Gérard Monfort Éditeur (Ed. Or, 1989). 
GALASSI Cristina, 2005, Sculture « da vestire ». Nero Alberti da Sansepolcro e la produzione di manichini lignei in una bottega del ‘500, Milano-Perugia, Electa-Editori Umbri Associati.

GELL Alfred, 2009, L'art et ses agents, une théorie anthropologique, Gand, Les Presses du réel (Ed. Or, 1998).

HEINICH Nathalie, 2009, La fabrique du patrimoine. De la cathédrale à la petite cuillère, Paris, Maison des sciences de l'homme, coll. «Ethnologie de la France ».

Jacquard. Pagine di cultura tessile, 2009, nov., $\mathrm{n}^{\circ}$ 64, Fondazione Lisio. Arte della seta, Nardini Editore.

L'Homme. Revue française d'anthropologie 2003. «Image et anthropologie », présentation Carlo Severi, $\mathrm{n}^{\circ} 165$, janv.-mars, Paris, Éditions de l'EHESS.

Refice Paola, Conticelli Valentina, GATTA Secondino (dir.), 2005, Madonnine agghindate. Figure devozionali vestite dal territorio di Arezzo, 2005, Soprintendenza per i beni architettonici e per il paesaggio e per il patrimonio storico, artistico ed etnoantropologico per la provincia di Arezzo.

SILVESTRINI Elisabetta e SimEONI Elisabetta (a cura di), 1987, n 16, oct-déc., « La cultura della bambola », La Ricerca Folklorica. Contributi allo studio della cultura delle classi popolari.

SILVESTRINI Elisabetta, 2010a, Simulacri vesti devozioni. Etnografia delle statue « da vestire » della provincia di Latine. Repertori dell'Arte del Lazio-1, dir. scientifica di Rossella Vodret, Soprintendenza per i Beni Storici, Artistici ed Etnoantropologici del Lazio, Roma, « L’Erma » di Bretschneider.

SILVESTRINI Elisabetta (a cura di), 2010b, oct. $\mathrm{n}^{\circ}$ 62, « Vestire i simulacri », Erreffe. La ricerca folklorica. Contributi allo studio della cultura delle classi popolari.

TREXLER Richard, 1991, « Habiller et déshabiller les images, esquisse d'une analyse » in Dunand F., Spieser J.-M., Wirth J., L'image et la production du sacré, Paris, Méridiens Klinsieck.

Violi Marco, BoRTolotTI Lidia, 2008, Virgo Mater Regina : percorsi per una conoscenza dei simulacri vestiti in Diocesi di Imola, Mostra, Imola, oratorio di San Rocco, 6-21 dicembre 2008, Circolo culturale « Luigi Einaudi », Castelfranco.

\section{NOTES}

1. Ce chiffre inclut les Vierges disparues. À l'époque de l'enquête, il existait encore vingt-sept Vierges habillées. Certaines étaient toujours offertes au culte, d'autres se trouvaient dans le musée diocésain.

2. B. Andriano Cestari (dir.), 1995 ; P. Refice, V. Conticelli, S. Gatta (dir.), 2005 ; C. Galassi, 2005 ;

M. Violi et L. Bortolotti, 2008 ; E. Silvestrini, 2010a.

3. J'extrais cette citation de la phrase par laquelle D. Freedberg ouvre son livre sur le pouvoir des images: «Ce livre ne traite pas d'histoire de l'art. Il traite des relations entre les images et les gens au cours de l'histoire. Il prend délibérément en compte toutes les images, et pas seulement celles que l'on considère comme artistiques " (1998, p. 9). Dans l'ouvrage, paru un an après, H. Belting prend une position similaire, avec cette différence, cependant, qu'il historicise la relation entre image et art. Son but, déclare-t-il, est d'écrire une « histoire de l'image », le temps des images de culte ayant précédé selon lui « l'ère de l'art » (1998, p. 5).

4. J'emprunte l'expression au titre du livre de N. Heinich sur les processus de patrimonialisation dans les sociétés contemporaines (2009). 
5. Je mets le terme entre guillemets pour indiquer que ces « statues " n'en sont pas vraiment. Nos collègues italiens les désignent fréquemment par le terme simulacro. En espagnol - et l'on peut y voir un signe de la diffusion de ce type de «statues " dans le monde hispanique - on dispose de plusieurs termes pour les nommer : imagen de candelero, imagen de bastidor, imagen vestidera.

6. Je me permets de signaler qu'un chapitre du livre que j'ai publié en 2002 sur les images miraculeuses de la Vierge porte sur cette question, que j'ai abordée à partir de terrains espagnols.

7. L'Espagne, et plus particulièrement l'Andalousie, offre un troisième cas de figure. Les statues processionnelles de la Semaine Sainte, celles de la Vierge en particulier, ainsi que leurs vêtements (absolument somptueux) et leurs parures sont investis d'une très grande valeur à la fois esthétique, religieuse et identitaire. Mais, à la différence de ce qui se produit en Italie, et peut-être parce que l'usage cultuel de ces statues est bien vivant, l'activité patrimoniale est prise en charge par les confréries, propriétaires de ces statues. Certaines ont une salle d'exposition permanente très analogue à un trésor (à Séville, la confrérie de la Vierge de la Macarena, par exemple, dispose d'une salle située dans l'église); d'autres se regroupent pour organiser des expositions temporaires (cela a été fait, dans les dernières années, à Lorca, Valdepeñas, Málaga) ou projettent d'ouvrir un musée pour y exposer de façon permanente leur patrimoine, ainsi à Cordoue et à Cadix.

8. R. Trexler publie un article sur la question en 1991. Mais cette étude s'appuie uniquement sur une documentation historique alors que R. Pagnozzato a fait des enquêtes auprès des habilleuses, ce qui lui permet de dégager le sens qu'elles donnent à cette opération.

9. J'ai fait la plupart de mes enquêtes en Espagne sans connaître celles de R. Pagnozzato. Elles concordent à un point étonnant: les femmes, ici comme là, parlent de leur expérience de l'habillage dans les mêmes termes et avec la même émotion. Il n'est pas sans intérêt de noter que les restauratrices des vêtements des Vierges évoquent également l'émotion qu'elles ont éprouvée en rhabillant la statue (voir les comptes rendus de restauration publiés dans la revue Jacquard $2009: 23,30,36)$.

10. Surtout pour nous qui connaissons les effets de la chimiothérapie sur la chevelure.

11. R. Pagnozzato (1993 : 106) souligne, quant à elle, que l'on fait une nette distinction entre les sculpteurs sur marbre ou sur bronze et les sculpteurs sur bois à partir du XVI ${ }^{\mathrm{e}}$ siècle.

12. Cf. Freedberg 1998 [1989]: "... les xoana sont censés provenir des cieux et cette croyance explique probablement les pouvoirs divins dont ils sont investis. Mais il se peut fort bien que leur aspect et le lieu de leur découverte aient eu une importance sous ce rapport. Ils apparaissent archaïques et austères et ces caractères, précisément, impressionnent et engagent à les diviniser $»$ (p. 49).

13. Soulignons, sur ce point, que ces «mutilations" (bras coupés et remplacés par des pièces amovibles ou articulées) ne peuvent être le fait du «peuple». Car ces opérations n'ont pu être faites sans que le curé ou les dirigeants de la confrérie chargée de veiller sur l'image aient donné leur aval. Il est même probable que le « peuple » n'ait pas été informé, dans la plupart des cas, des transformations apportées à la statue.

14. Il faudrait y ajouter, notamment, les relectures de l'œuvre d'Aby Warburg. On lira, sur ce point, le dossier sur « Image et anthropologie » présenté par C. Severi dans L'Homme, 2003. 


\section{AUTEUR}

\section{MARLÈNE ALBERT LLORCA}

LISST-Centre d'Anthropologie Sociale, Université de Toulouse-Le Mirail, marlene.albert@wanadoo.fr 